\title{
Diagnóstico e intervenção em empresas médias: uma proposta de (re)organização das atividades do Processo de Desenvolvimento de Produtos
}

\author{
Márcia Elisa Echeveste ${ }^{a, *}$, José Luis Duarte Ribeiro ${ }^{b}$ \\ a,*echeveste@producao.ufrgs.br, UFRGS, Brasil \\ bribeiro@producao.ufrgs.br, UFRGS, Brasil
}

\begin{abstract}
Resumo
O Processo de Desenvolvimento de Produtos (PDP) pode ser definido como a maneira pela qual uma empresa converte ideias e oportunidades em produtos e serviços. Esse processo exige etapas bem definidas e administração de pessoas e recursos. Nos últimos anos, surgiram modelos referenciais de desenvolvimento que se aperfeiçoaram e incorporaram novas fases no desenvolvimento de produtos, desde a análise do mercado até a descontinuidade do produto. Apesar disso, no que se refere à estruturação do PDP, de um modo geral, as empresas, sobretudo pequenas e médias, não adaptaram facilmente à sua realidade os modelos defendidos nos livros. Claramente, falta uma função de transição entre a prática atual e as propostas teóricas. Baseado na literatura e na prática observada em empresas, este artigo apresenta um método de estruturação das atividades concernentes ao PDP de empresas de bens de manufatura. A proposta contempla a apresentação de um método utilizado para melhoria da integração das atividades. Os métodos utilizados mostram como, na prática, pode ser feita a intervenção em uma empresa de médio porte que deseja organizar seu Processo de Desenvolvimento de Produtos.
\end{abstract}

Palavras-chave

Engenharia de produção. Desenvolvimento de produtos. Integração funcional.

\section{Introdução}

Uma retrospectiva histórica revela que os aspectos que caracterizaram o desenvolvimento de produtos nas décadas passadas foram ciclos mais longos, departamentos com funções individualizadas, com etapas executadas de forma sequencial. Por outro lado, na década atual, muitas empresas estão migrando para o desenvolvimento de ciclos menores, multifuncionalidade e integração entre departamentos. Observa-se que as fases do desenvolvimento de produtos tornaram-se simultâneas, em resposta à necessidade de diminuir os ciclos de desenvolvimento e inovação.

Para Clift e Vandenbosh (1999), um dos principais objetivos do desenvolvimento de novos produtos é a redução do tempo do ciclo. Para atingir esse objetivo, é necessário o gerenciamento de equipes multifuncionais (COOPER; KLEINSCHIMST, 1994;
PRASAD, 1997); o envolvimento do consumidor (GRIFFIN; PAGE, 1993; GRUNER; HOMBURG, 2000) e o planejamento dos mecanismos de comunicação junto à equipe de desenvolvimento (WHEELWRIGHT; CLARK, 1992). Esses elementos contribuem para a melhor organização do processo interno de desenvolvimento.

Conforme Cooper (1994), é reconhecido que diminuir tempos de desenvolvimento, responder rapidamente às mudanças e descobertas tecnológicas, instituir mecanismos eficientes de comunicação interna e estabelecer estruturas formais de desenvolvimento são fatores que contribuem para o sucesso de novos produtos. Duas diretrizes importantes estão por trás desses fatores: reconhecer o Processo de Desenvolvimento de Produto (PDP) como um processo de negócio 
com forte orientação para o mercado e a existência de mecanismos formais de estruturação e melhoria do PDP.

No entanto, não são todas as empresas que estão trabalhando de acordo com os preceitos do desenvolvimento integrado, em que as áreas envolvidas deveriam estar organizadas em uma equipe de projeto. Principalmente, algumas empresas de médio porte estão buscando formas mais eficientes de gerenciamento de PDP, com maior envolvimento interdepartamental. Caracteriza-se neste trabalho uma empresa de médio porte como aquelas que têm entre 100 e 499 empregados (SERVIÇO..., 1999). A proposta deste trabalho é para empresas de manufatura de bens e serviços cujo PDP tem grau de maturidade anterior à padronização. Assim, seus processos de desenvolvimento dependem da memória das pessoas envolvidas e da repetição das atividades. Normalmente, essas empresas são provenientes de uma organização familiar e possuem poucos envolvidos no PDP. Os projetos são coordenados por um representante de engenharia que tem contato direto com o departamento comercial e com pessoas-chave dos departamentos de produção e vendas. Muitas vezes o departamento de marketing não existe formalmente, fazendo com que os representantes de vendas sejam responsáveis por trazerem as demandas dos clientes.

Segundo pesquisa realizada por Fonseca e Kruglianskas (2002), em incubadoras de São Paulo, no que tange à inovação, as empresas brasileiras tradicionais apresentam condições financeiras mais reduzidas para arcar com os custos de modernização e de inovação tecnológica, não possuem cultura de inovação e têm dificuldades históricas de relacionamento com os ambientes de pesquisa. Da mesma maneira, a maioria das organizações, sobretudo as pequenas e médias empresas, têm seus processos não documentados e não estruturados; assim, o desenvolvimento e as práticas de inovação são conduzidos de forma desordenada, com conhecimento insuficiente do caminho a ser trilhado.

Essas empresas têm muitas vantagens em organizar seus processos. A estruturação do PDP pode melhorar o entendimento das demandas nas fases iniciais do desenvolvimento, diminuir o retrabalho de engenharia e facilitar o controle de custos, qualidade e tempo durante o desenvolvimento. Além disso, organiza a participação das diferentes áreas de conhecimento, as quais deixam de depender exclusivamente do conhecimento individual dos envolvidos.
Destaca-se que esse cenário do PDP é frequentemente observado nas empresas de porte médio que necessitam reorganizar seu processo de desenvolvimento. Essa constatação parte de entrevistas conduzidas pelos autores nas quais o objetivo foi entender como a empresa realiza o desenvolvimento de produto e quais as principais situações de conflito de acordo com a percepção dos departamentos envolvidos. Com base nessa observação, os autores compilaram os problemas reincidentes nesse tipo de empresa. Estes problemas foram: (i) falta de definições estratégicas no início do processo de desenvolvimento; (ii) falta de foco no negócio; (iii) falta da presença de investidores, acionistas e alta gerência nas fases de planejamento, em que as decisões estratégicas são tomadas somente por técnicos; (iv) falta de gerenciamento de equipes entre funções e intrafunções; (v) falta ou inadequação de recursos humanos que preenchem diversas funções; (vi) falta de definição do processo de desenvolvimento na forma de uma sequência lógica de etapas e de atividades, que devem ser documentadas, disseminadas e entendidas por todos membros da empresa; (vii) falta de conhecimentos de ferramentas aplicadas ao desenvolvimento das atividades do PDP; (viii) foco do desenvolvimento exclusivamente no projetista ou no departamento de desenvolvimento de produtos; (ix) falta de uma política de gestão de portifólio, priorização e acompanhamento financeiro de projetos.

Nos últimos anos, modelos de desenvolvimento foram aperfeiçoados e adequados à nova realidade. Porém, a ampliação do PDP, incluindo novas áreas de conhecimento, exigiu novos esforços teóricos. Nesse sentido, destacam-se os modelos propostos por Roozenburg e Eekels (1995), Crawford e Di Benedetto (2000), e no Brasil, modelos referenciais que organizaram boas práticas de gestão, como Rozenfeld et al. (2006).

Nos livros que tratam de Desenvolvimento Integrado de Produtos (DIP) e melhorias no PDP, as constatações são lógicas e soam como orientações óbvias. No entanto, um guia factível para a condução do PDP à realidade de empresas de menor porte ainda é uma meta não atingida. Empresas maiores, que seguem modelos de desenvolvimentos da empresa matriz, reclamam da falta de adequação da teoria à realidade brasileira. Entender como ocorre a integração entre as áreas é uma forma de viabilizar o processo de melhoria contínua e estabelecer as mudanças.

Essa temática de mudanças vem sendo discutida em periódicos e livros mais atuais. Alguns autores discutiram modelos de intenvenção; entre eles, destacam-se Rentes (2000), Wood Junior (2002) 
e Bressan (2004), que apresentaram modelos de intervenção para melhoria contínua em processos baseados nos conceitos de gestão da mudança. McAdam, Stevenson e Armstrong (2000) utilizaram a abordagem de intervenção similar em melhoria de processos de manufatura. Por sua vez, mais recentemente, Rozenfeld et al. (2006) resumiram as melhores práticas de implantação de modelos referenciais do PDP em um modelo denominado pelos autores de "modelo de transformação" do PDP.

Os estudiosos sabem onde as empresas deveriam chegar, e algumas delas sabem o que deveria ser feito, mas o caminho de transição entre a situação atual e a futura não é claro. Como operacionalizar essas mudanças e trilhar o caminho até a organização das atividades de desenvolvimento de forma integrada, interfuncional, reduzindo tempo de desenvolvimento e, mais que isso, estabelecendo um processo de desenvolvimento orientado ao mercado, considerando as potencialidades internas, é um desafio para a maioria das empresas.

0 Processo de Desenvolvimento de Produtos é o modo pelo qual os novos são desenvolvidos desde a identificação de uma necessidade do mercado em um produto ou serviço tangível (PHILLIPS; NEAILEY; BROUGHTON, 1999). Esse processo não é de fácil gerenciamento, pois incorpora numerosas atividades e quase todos os departamentos da empresa.

Este artigo apresenta uma proposta para a estruturação do PDP direcionada a empresas de médio porte que ainda não possuem estruturas formais de desenvolvimento de produto. Trata-se da apresentação de um método de estruturação e organização das atividades baseado na análise teórica dos modelos propostos por Andreasen e Hein (1987), Baxter (1998), Cooper (1994), Crawford e Di Benedetto (2000), Cunha (2003), Kotler (1998), Pahl e Beitz (1996) e Roozenburg e Eeekels (1995), bem como na prática observada em empresas sediadas na região Sul do Brasil.

Em relação à proposta deste artigo, ressalta-se a aplicabilidade e facilidade que ela proporciona na condução da integração das atividades à realidade da empresa. Vale mencionar que pode ser implementada por pessoas internas à organização. A proposta deste artigo está na estruturação do PDP, baseada nos conceitos de integração interfuncional e participação da alta direção em momentos críticos do processo de desenvolvimento. Este tipo de empresa se caracteriza por uma forte centralização nas decisões, muitas vezes provenientes do proprietário. Nesse tipo de empresa, o "departamento" de P\&D costuma ser constituído por 6 a 10 pessoas, e os projetos de desenvolvimento são realizados por uma mesma equipe. A disponibilidade de recursos humanos é restrita. Os modelos de tranformação no PDP disponíveis na literatura mencionada foram criados para empresas com maior capacidade de recursos humanos, nas quais é possível trabalhar com diferentes equipes de desenvolvimento em múltiplos projetos simultâneos.

A proposta de estruturação está subdividida em uma fase de intervenção no PDP e, na sequência, propõe-se um método de integração das atividades. A intervenção no PDP compreende as etapas de caracterização da empresa, diagnóstico da situação atual e proposta de melhorias. Dentre a proposta de melhorias está o modelo de integração de atividades.

\section{Metodologia de pesquisa}

0 método de intervenção proposto é resultado da revisão da literatura e realização de uma pesquisa exploratória em empresas de bens de manufatura, de grande, médio e pequeno porte, situadas na região Sul do Brasil. A escolha das indústrias foi realizada por julgamento. As empresas de grande porte são consideradas centros de excelência em desenvolvimento de produtos. As de médio porte têm organização predominantemente familiar, tiveram seu faturamento ampliado nos últimos anos, contudo ainda se caracterizam por baixo nível de maturidade na organização de seus processos, sobretudo de desenvolvimento de produtos.

Inicialmente, realizou-se a exploração inicial do tema tomando como base a prática das empresas estudadas, através de entrevistas em profundidade conduzidas pelos autores deste artigo. Uma descrição geral das características dessas empresas aparece no Quadro 1.

Após a exploração inicial, durante um ano, realizou-se uma imersão numa empresa de porte médio com o propósito de implementar o modelo referencial de desenvolvimento de produtos. Durante esse período, foi possível, na condição de observador participante, acompanhar o processo de intervenção.

Apoiado no estudo da literatura e no aprendizado junto a essas indústrias, foi estabelecido um método de intervenção para auxiliar médias empresas a implantarem um modelo de estruturação das atividades do PDP segundo os preceitos da gestão de Desenvolvimento Integrado de Produtos. 0 modelo inclui a descrição das melhores práticas vivienciadas, enunciando os procedimentos 
Quadro 1. Descrição das empresas pesquisadas.

\begin{tabular}{|c|c|c|c|}
\hline Ramo da empresa & Entrevistado & $\begin{array}{l}\text { Porte da } \\
\text { empresa }\end{array}$ & Situação do PDP \\
\hline Automotivo & $\begin{array}{l}\text { Engenheiro de } \\
\text { produto }\end{array}$ & Grande & $\begin{array}{l}\text { PDP sem estruturação, documentos dispersos entre os departamentos. } \\
\text { Grande responsabilidade do projetista. Falta de comunicação entre } \\
\text { marketing, produção e projeto. }\end{array}$ \\
\hline Eletroeletrônico & $\begin{array}{l}\text { Coordenador } \\
\text { de projeto } \\
\text { Gerente de } \\
\text { marketing }\end{array}$ & Grande & $\begin{array}{l}\text { PDP estruturado, ajustes no processo atual de desenvolvimento, fase } \\
\text { de otimização do processo de desenvolvimento para obter melhores } \\
\text { resultados, dificuldades de integração interfuncional. }\end{array}$ \\
\hline Brinquedos & $\begin{array}{l}\text { Gerente geral } \\
\text { Engenheiro de } \\
\text { produto }\end{array}$ & Médio & $\begin{array}{l}\text { PDP não estruturado, falta de controle sobre tempo, custos e qualidade } \\
\text { do projeto. Etapas executadas de maneira informal, sem conhecimento } \\
\text { da ordem. Falta de hierarquia de decisão. }\end{array}$ \\
\hline Componentes & Gerente geral & Pequeno & $\begin{array}{l}\text { PDP em fase de estruturação e documentação, desenvolvimento } \\
\text { sequencial migrando para desenvolvimento integrado. }\end{array}$ \\
\hline $\begin{array}{l}\text { Equipamentos } \\
\text { para armazenagem } \\
\text { e transporte }\end{array}$ & $\begin{array}{l}\text { Engenheiro de } \\
\text { produto }\end{array}$ & Grande & $\begin{array}{l}\text { Falta de processos de desenvolvimento estruturados, grande dependência } \\
\text { da memória e capacidade técnica das pessoas envolvidas. }\end{array}$ \\
\hline Máquinas agrícolas & $\begin{array}{l}\text { Engenheiro de } \\
\text { produto }\end{array}$ & Pequeno & $\begin{array}{l}\text { Os problemas de pós-venda que poderiam ser resolvidos no projeto } \\
\text { motivaram a empresa a investir no desenvolvimento de produtos. PDP } \\
\text { não estruturado, empresa procura desenvolver modelo referencial e } \\
\text { padronização do PDP. }\end{array}$ \\
\hline Aeronáutica & Engenharia & Grande & $\begin{array}{l}\text { PDP definido, padronizado, fase de otimização do processo e compilação } \\
\text { de métricas. Fase de estabelecimento de melhorias na capacidade de } \\
\text { integração entre áreas e de indicadores durante o PDP. }\end{array}$ \\
\hline
\end{tabular}

adotados que levaram ao caminho de sucesso na implantação.

Ressalta-se que o envolvimento em estudos práticos, no início da exploração do tema (empresas de grande, médio e pequeno porte), contribuiu para o aprendizado e desenvolvimento do método e, ao final, como forma de validação parcial da proposta de estruturação.

Com base nos estudos de caso, procedeu-se o aprimoramento dos procedimentos propostos, de forma a tornar a abordagem mais abrangente e útil para um número maior de segmentos industriais. As próximas seções apresentam a versão final dos procedimentos que compõem o método de intervenção e integração do PDP.

\section{Apresentação do método de estruturação do PDP}

A Figura 1 sintetiza a proposta de estruturação do PDP, destacando alguns aspectos a serem analisados em seu processo de implementação. 0 método de intervenção é indicado a empresas que não têm seus processos suficientemente documentados, enquanto o método de integração das atividades oferece uma abordagem para operacionalizar a integração entre departamentos e atividades do desenvolvimento conjunto de produtos.

\subsection{Caracterização do ambiente de negócio}

Modelos referenciais do PDP, que descrevem o sequenciamento das atividades, tarefas e pessoas envolvidas, podem variar dependendo da empresa e do tipo de produto. Contudo, Phillips, Neailey e Broughton (1999) e Wheelwright e Clark (1992) compararam grandes empresas quanto às etapas de desenvolvimento e concluíram que suas macroatividades são similares. 0 importante é entender como as etapas serão gerenciadas e como elas se relacionam entre as diferentes áreas ou departamentos. Esse entendimento é decorrente da cultura da empresa e das características do ambiente de negócio no qual está inserida.

0 entendimento a priori dessas características permite uma adequação do PDP à cultura e à linguagem da empresa. É preciso estabelecer um caminho viável entre a situação atual e a mudança que se deseja estabelecer. Alguns elementos podem ser considerados nesta fase. Por exemplo, a posição da concorrência e os fatores externos, tais como influência socioeconômica, leis governamentais, fiscais e normalizações. Além deles, também devem ser levadas em conta as características internas, como natureza do mercado e dos canais de distribuição, investimentos em recursos de desenvolvimento e a importância atribuída ao desenvolvimento de novos produtos. Outros aspectos são igualmente 


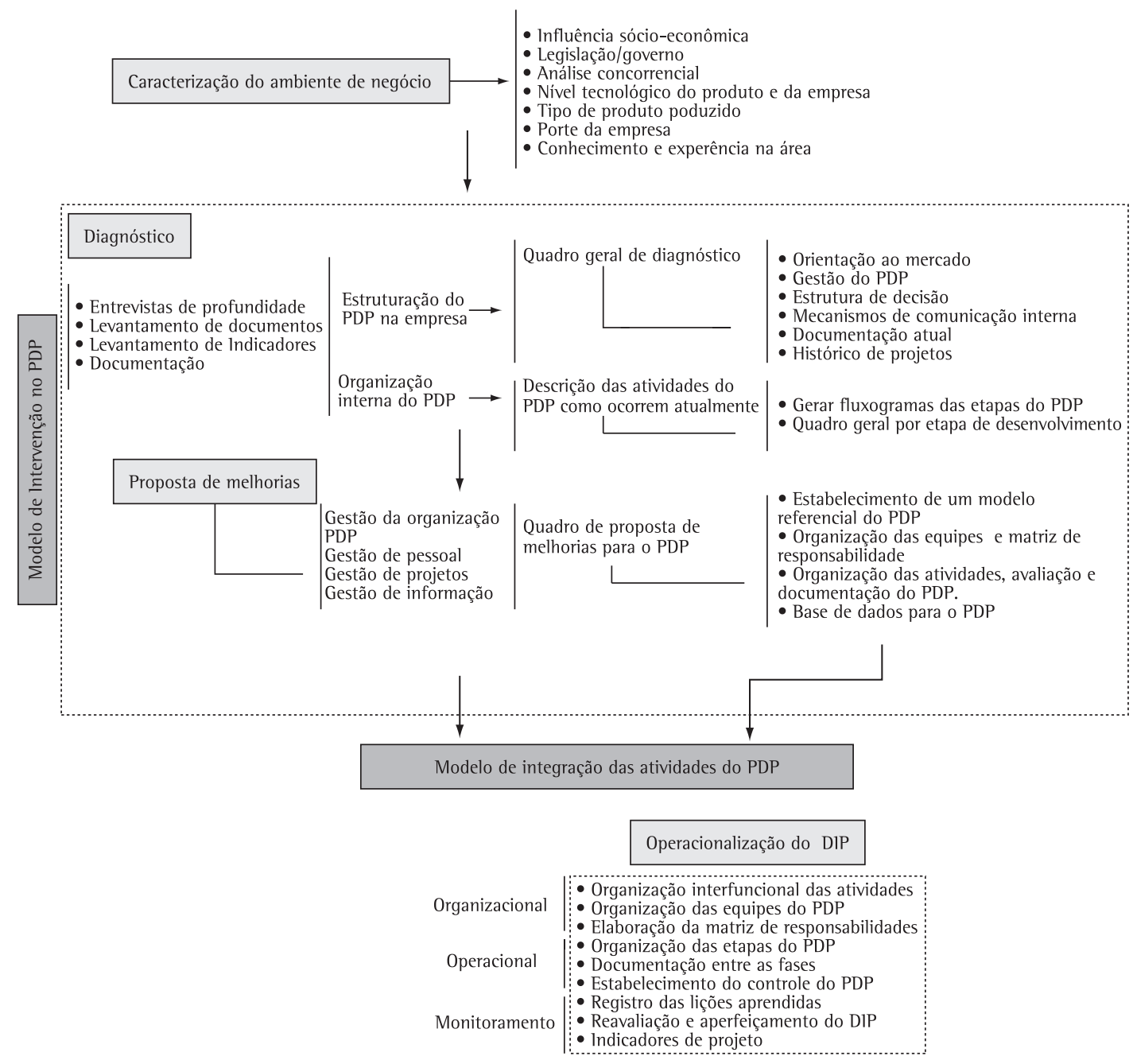

Figura 1. Modelo de estruturação do PDP. Fonte: elaborado pelos autores.

importantes para a caracterização do ambiente, como capacidade de inovação da empresas, frequência de lançamentos de novos produtos em relação ao praticado na concorrência, integração entre os departamentos de marketing, projeto, produção e engenharia.

\subsection{Método de intervenção no PDP}

A intervenção no PDP parte de um diagnóstico da situação atual praticada na empresa de acordo com a sua cultura e procedimentos realizados no desenvolvimento de produtos, seja de modo formal ou informalmente. A prática atual é modelada, por meio de fluxogramas que representam mapas com demonstração das fases do PDP, com as áreas envolvidas, e comparada com um modelo referencial a ser adotado pela empresa. Alguns modelos retratam a manufatura, destacando a proposta de Rozenfeld et al. (2006). Com base num modelo referencial, o próximo passo é a implementação gradual das atividades do modelo referencial ao modelo atual praticado na empresa.

\subsubsection{Diagnóstico da situação atual}

0 diagnóstico refere-se a um detalhamento da situação atual do PDP para o levantamento das situações de conflito e de como é realizado o PDP. Em outras palavras, a forma como o processo de desenvolvimento é executado e a caracterização do ambiente de negócio são fundamentais para estabelecer uma análise da situação atual e, a partir dessa análise, definir um caminho de transição visando a estruturação do PDP. 
Para realizar o diagnóstico, podem ser utilizadas entrevistas em profundidade, contemplando representantes da direção, vendas, marketing, produção, engenharia, projetos e compras. 0 objetivo dessa fase é o conhecimento da relação de cada departamento com o PDP, das dificuldades, segundo a ótica dos departamentos, referentes ao processo de desenvolvimento praticado pela empresa e, finalmente, o levantamento da experiência de cada um em relação a desenvolvimentos anteriores. Procuram-se razões que poderiam explicar o sucesso, ou não, do processo de desenvolvimento. Paralelamente às entrevistas, o acompanhamento de reuniões e a análise da documentação e de indicadores relacionados ao desenvolvimento de produto constituem outras fontes de informação.

A fase de diagnóstico tem como objetivo conhecer como o departamento responsável pelo desenvolvimento de produto se relaciona com os demais setores da empresa e saber como a empresa gerencia o PDP. Para organizar as informações provenientes das entrevistas e da análise da documentação, sugere-se o preenchimento de um "quadro geral de diagnóstico", o qual apresenta os resultados de forma resumida, de acordo com tópicos de interesse: como a empresa procede a orientação para o mercado e desenvolve os planos de marketing; como realiza atualmente a gestão de processo de desenvolvimento; qual a estrutura de decisão orientada à execução das atividades do PDP; como funcionam na prática os mecanismos de comunicação interna; quais os documentos existentes que fornecem base de informação ao PDP e, finalmente, qual seria o histórico dos projetos, com o objetivo de investigar se há registro de projetos anteriores e como foram executados.

Concomitante ao entendimento da estruturação da empresa, investiga-se a organização interna do PDP, independentemente do nível de estruturação existente. Como produto, tem-se a geração dos mapas do processo atual de desenvolvimento e a organização de um quadro resumindo como são executadas as fases de desenvolvimento de produto. Esse quadro, exemplificado na Figura 2, apresenta as fases relacionadas aos principais pontos de conflitos. As fases foram agrupadas por área ou atividade do PDP.

\subsubsection{Proposta de melhorias}

Baseado no diagnóstico, propõe-se algumas ações de transição que poderão ser executadas a curto e médio prazo. Clark e Fujimoto (1991) descrevem três dimensões de organização do desenvolvimento de produto: preservação do conhecimento tecnológico e eficiência das tarefas individuais; integração interna (coordenação de tarefas) e integração externa ou tradução do conceito do produto às exigências do consumidor. A próxima seção descreve uma proposta de um modelo de integração das atividades internas.

Segundo Crow (2002), para caracterização do PDP e documentação do Processo de Desenvolvimento de Produto devem ser considerados os seguintes

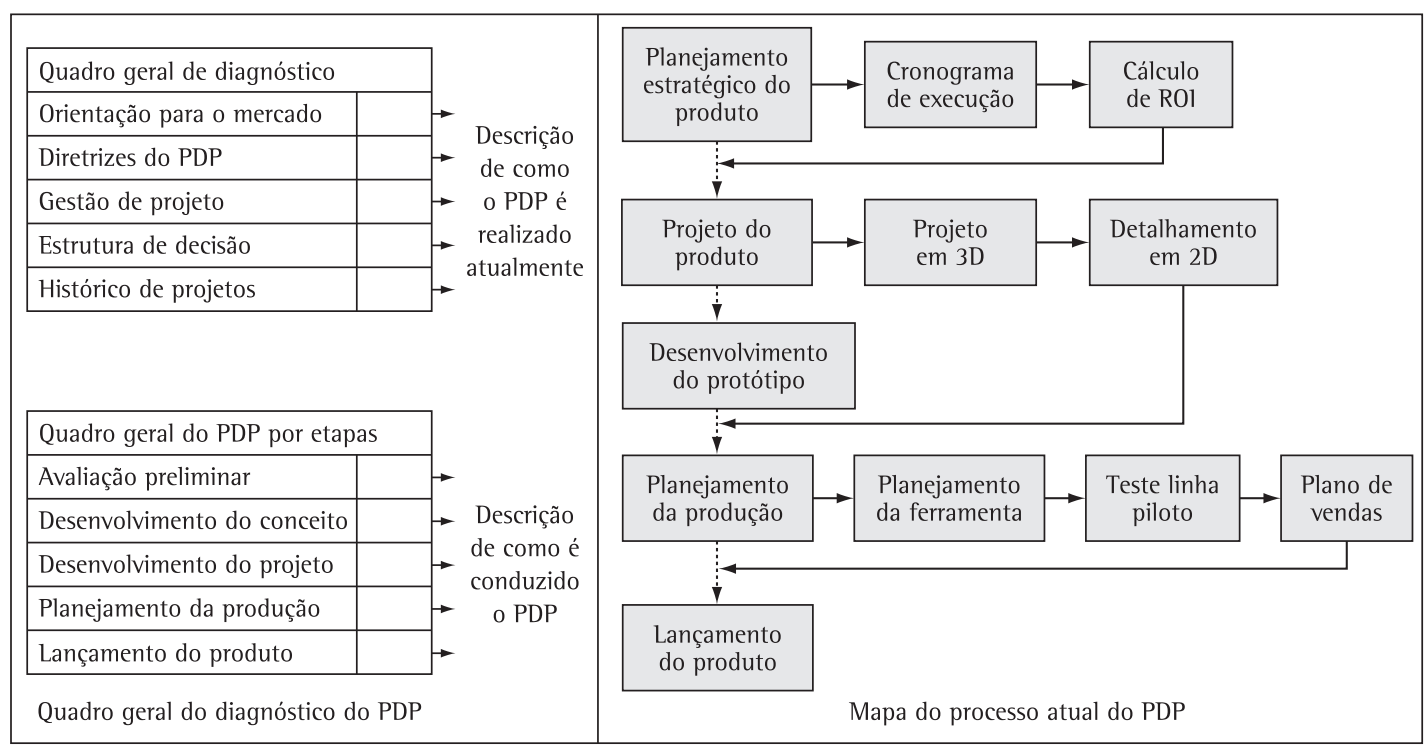

Figura 2. Apresentação dos resultados do diagnóstico do PDP. Fonte: elaborada pelos autores. 
elementos: (1) fluxograma do processo; (2) descrição das etapas incluindo inputs e resultados esperados, responsabilidades e ferramentas de suporte; (3) descrição de cada etapa comparada com o padrão previamente exigido; (4) descrição do processo de revisão de fases e revisão através de marcos de revisão de fases (stage-gate ${ }^{\circledR}$ ), incluindo participantes, informação necessária e programação das reuniões e (5) critérios para construção de protótipos.

Exemplos de propostas de melhorias face às oportunidades existentes aparecem resumidamente no Quadro 2. Conforme o quadro, o primeiro passo para a melhoria é o estabelecimento de um modelo referencial de integraçao das atividades. Esse modelo refere-se a uma adaptação dos modelos de referência encontrados na literatura (PAHL; BEITZ, 1996; CRAWFORD; DI BENEDETTO, 2000; ROZENFELD et al., 2006). Com base no modelo escolhido, a equipe de intervenção estabelece quais atividades e em que ordem poderiam ser implementadas para a empresa. 0 Quadro 2 sugere um ordenamento das atividades a serem executadas que podem ser lidas nas colunas do quadro. Inicialmente a gestão do PDP refere-se à escolha do modelo referencial a ser adotado e a forma de gestão. Neste caso, a forma de gestão é o Desenvolvimento Integrado de Produtos (DIP). A gestão de pessoas pressupõe a organização de equipes interfuncionais. A (re)organização das atividades utiliza os princípios de gestão de projetos que compreende a organização das atividades de execução, seguidas de atividades de controle e documentação. Ao final, após o planejamento desses passos, empregam-se os conceitos da gestão do conhecimento, que abrange a gestão da informação e capacitação dos envolvidos para projetos futuros.

0 método de integração das atividades do PDP está apresentado na Figura 3. Ele segue os preceitos do DIP, como preocupações de integração entre áreas (marketing, engenharia, manufatura, finanças, compras etc.) com o objetivo de desenvolver produtos (ANDREASEN; HEIN, 1987). 0 DIP prega o paralelismo na execução das atividades que, aplicado ao PDP, emprega equipes de diferentes funções para fabricar produtos que atendam efetivamente ao mercado.

De forma geral, o PDP constitui-se de três grandes fases: (i) o pré-desenvolvimento, que tem como evento principal as diretrizes de lançamento de novos produtos e a geração do conceito que atende à oportunidade de negócio; (ii) o desenvolvimento propriamente dito, que abrange a elaboração do conceito, o projeto preliminar e detalhado do produto, planejamento de marketing e da produção, desenvolvimento da produção e liberação para vendas; e (iii) o pós-desenvolvimento, envolvendo avaliação e registro de dados (indicadores de acompanhamento do projeto) e lições aprendidas (registro de práticas que atualizem e aperfeiçoem

Quadro 2. Proposta de mudanças no PDP.

\begin{tabular}{|c|c|c|}
\hline \multicolumn{2}{|r|}{ Área } & Proposta \\
\hline $\begin{array}{l}\text { Gestão do } \\
\text { PDP }\end{array}$ & $\begin{array}{l}\text { Desenvolvimento } \\
\text { Integrado de Produtos }\end{array}$ & $\begin{array}{l}\text { Estabelecer um modelo de integração das atividades de Desenvolvimento Integrado } \\
\text { de Produtos (DIP) adaptado às características da empresa. }\end{array}$ \\
\hline \multirow{2}{*}{$\begin{array}{l}\text { Gestão de } \\
\text { pessoal }\end{array}$} & $\begin{array}{l}\text { Definir equipes } \\
\text { interfuncionais }\end{array}$ & $\begin{array}{l}\text { Definir uma política de organização da equipe de projeto; } \\
\text { Criar uma comissão de avaliadores das fases intermediárias do desenvolvimento de } \\
\text { produtos. }\end{array}$ \\
\hline & $\begin{array}{l}\text { Matriz de } \\
\text { responsabilidades }\end{array}$ & Estabelecer uma matriz de responsabilidades voltada ao PDP. \\
\hline \multirow{3}{*}{$\begin{array}{l}\text { Gestão de } \\
\text { projetos }\end{array}$} & $\begin{array}{l}\text { Organização das } \\
\text { atividades }\end{array}$ & $\begin{array}{l}\text { Estruturar as atividades do PDP; } \\
\text { Gerar um cronograma geral de projetos com auxílio de softwares; } \\
\text { Gerar um fluxograma do desenvolvimento de produto; } \\
\text { Organizar as atividades por departamento e por fase de desenvolvimento. }\end{array}$ \\
\hline & Avaliação & $\begin{array}{l}\text { Definir os pontos de revisão do processo de desenvolvimento e de avaliação do PDP; } \\
\text { Estabelecer critérios de avaliação conceitual, de protótipo e ferramental; } \\
\text { Determinar indicadores de projeto e registro de experiências aprendidas. }\end{array}$ \\
\hline & Documentação & $\begin{array}{l}\text { Documentação das macroatividades do desenvolvimento; } \\
\text { Formalização de documentos que registrem as decisões do PDP. }\end{array}$ \\
\hline \multirow[t]{2}{*}{$\begin{array}{c}\text { Gestão do } \\
\text { conhecimento }\end{array}$} & $\begin{array}{l}\text { Gerenciamento } \\
\text { da informação }\end{array}$ & $\begin{array}{l}\text { ldentificar todas as informações que deveriam estar disponíveis antes e durante o PDP; } \\
\text { Criar uma base de informações integrada, para suporte aos departamentos; } \\
\text { Gerar um dossiê por projeto, com documentação de todas as fases, tempos de } \\
\text { desenvolvimento e custos. }\end{array}$ \\
\hline & $\begin{array}{l}\text { Capacitação } \\
\text { pessoal }\end{array}$ & $\begin{array}{l}\text { Estabelecer treinamento específico para líderes de projeto e equipe de projeto. } \\
\text { Estipular programas de treinamento que visem melhorias no PDP. }\end{array}$ \\
\hline
\end{tabular}

Fonte: elaborada pelos autores. 


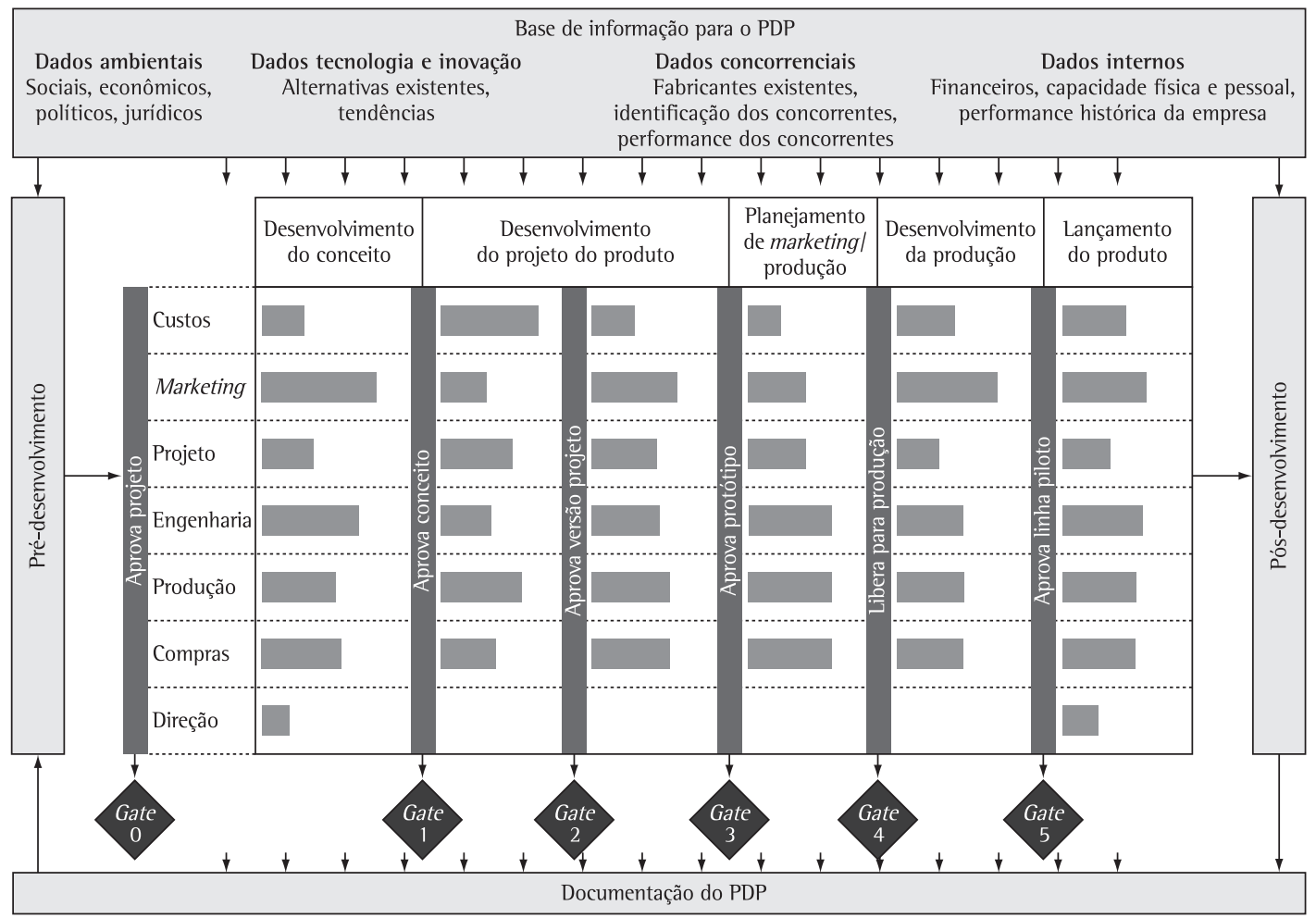

Figura 3. Modelo de integração das atividades do PDP. Fonte: elaborada pelos autores.

o modelo). 0 método utilizado de integração das atividades, proposto na próxima seção, apresenta a organização das atividades para a fase de desenvolvimento.

\section{Método de integração da atividades}

\subsection{Organização interfuncional das atividades}

Conforme mencionado, o Processo de Desenvolvimento de Produtos envolve atividades intradepartamentais e interdepartamentais. A cada ponto crítico (gate), a equipe envolvida é responsável por avaliar as atividades até aquela fase e dar o sinal verde para as fases seguintes. No mínimo duas equipes poderão ser formadas: uma equipe operacional, com poder de execução e de avaliação das tarefas do PDP, e uma equipe gerencial, com poder de decisão, capaz de abortar ou redirecionar o projeto, podendo ser chamada de comissão executiva.

De acordo com a Figura 3, o processo está subdividido horizontalmente pelos departamentos envolvidos (linhas) e verticalmente pelas fases do PDP (colunas). 0 modelo tem como suporte a informação de diferentes fontes: dados do ambiente de negócio, dados da concorrência, dados de informação internos e dados de tecnologia e inovação que constituem a base de informação para o PDP. Construir essa base tem como fim auxiliar a equipe na tomada de decisões e fornecer suporte a possíveis modificações e adequações do produto durante o processo de desenvolvimento.

A próxima etapa da implementação envolve identificar qual informação deve estar disponivel para melhor fundamentar e conduzir o PDP. Por sua vez, cada informação gerada durante a execução da fase, ou que sirva de subsídio para as fases posteriores, é guardada, formando a documentação e a memória do PDP.

As fases do PDP são subdivididas por marcos de aprovação (gates) dispostos ao longo do processo. Esses marcos representam momentos críticos do processo, capazes de corrigir a trajetória, cancelar ou paralisar o projeto. Na lógica dos gates, a conclusão de cada fase é geralmente marcada por uma revisão/ avaliação do desempenho do projeto, tendo em vista responder se deve prosseguir para a próxima fase. As revisões no fim de cada fase são chamadas comumente por saídas de fase, passagens de estágio (stage gate ${ }^{\circledR}$ ) ou pontos de término (kill points). Cada fase, normalmente, inclui um conjunto de resultados 
esperados (entregas), definidos com o objetivo de estabelecer o controle e acompanhamento das atividades. Os documentos de passagem geralmente são dados por levantamentos de necessidades, desenhos ou especificações (projeto), manutenção (turn over) e outros (PROJECT..., 2000).

Na Figura 3, aparecem cinco gates ou marcos de revisão do processo, que se referem a pontos críticos do projeto, nos quais deve ocorrer a avaliação da qualidade do produto em desenvolvimento (custo e qualidade) e da qualidade do projeto (tempo e custo de desenvolvimento). São ilustrados no modelo de integração: o gate 0, para aprovação dos projetos; o gate 1, para aprovação do conceito do produto; o gate 2, para aprovação da versão do projeto do produto; o gate 3, para aprovação do protótipo; o gate 4, para liberação da produção e para aprovação da linha piloto e, finalmente, o gate 5, para aprovação do produto (liberação para vendas). A escolha dos gates pode variar de empresa para empresa, pois a sua localização depende da definição de quais pontos do projeto são considerados críticos e onde é possível detectar e corrigir possíveis desvios do planejado.

Os gates, inicialmente, devem ser caracterizados através de documentos de avaliação que constituem o passaporte para a continuidade do desenvolvimento, liberando as atividades subsequentes. Todo o projeto deve ter um acompanhamento dos documentos gerados e dos desvios das metas de projeto e de produto.

Os envolvidos em cada fase de desenvolvimento estão sinalizados com uma flecha que indica sua participação na avaliação daquela etapa. As barras paralelas que aparecem na Figura 3 representam sobreposição de atividades. Conforme o modelo, em todas as atividades de desenvolvimento os departamentos estão envolvidos em maior ou menor intensidade. 0 comprimento da barra representa a duração da atividade. Existem algumas que são dependentes, cujosucessorelaciona-seaocumprimento de uma atividade anterior. Outras, são independentes, podendo ser iniciadas depois de determinadas decisões de continuidade do desenvolvimento.

A Figura 4 apresenta um exemplo de organização interfuncional das atividades no PDP. 0 maior desafio do modelo de organização interfuncional das atividades é estabelecer uma forma lógica das etapas se inter-relacionarem e de serem conduzidas durante o processo de desenvolvimento, de forma a acelerá-lo, sem perder de vista a qualidade do produto a ser desenvolvido. A qualidade se refere a como o produto atende o mercado e as exigências de custo, que são os elementos determinantes da competitividade no mercado.

\subsection{Organização das equipes do PDP}

Como regra geral na organização de equipes, devem ser atendidos: (i) uma definição das competências e responsabilidades de cada departamento do PDP; (ii) os caminhos de decisão curtos e (iii) o comprometimento dos membros da equipe com o desenvolvimento do produto aprovado como viável (STARBEK; GRUM, 2001).

Para empresas de maior porte, que gerenciam o desenvolvimento através de gates, trabalha-se, no mínimo, com três equipes. Uma equipe para liberação dos gates, uma de revisores e a executora das atividades. A equipe de liberação dos gates (denominados "gatekeepers" por COOPER, 1994) está em posição hierárquica superior à de projeto, sendo responsável pela liberação de recursos na aprovação da viabilidade do projeto e pela liberação de capital durante o desenvolvimento. Como equipe de apoio, algumas empresas adotam a de revisores do projeto, constituída por pessoas da mesma hierarquia da equipe de projeto, que auxiliam na revisão de documentos importantes durante a execução do projeto, e a equipe de projetos propriamente dita, que executa as tarefas do PDP.

\subsection{Elaboração da matriz de responsabilidades}

Para gerenciar a organização das equipes, propõe-se uma matriz de responsabilidades exemplificada no Quadro 3. Conforme a matriz, as etapas de desenvolvimento foram cruzadas com os setores, no qual se estabelece uma política de organização das equipes. Vale mencionar que os setores estão envolvidos em todas as etapas do PDP. Contudo, cada setor está relacionado mais fortemente com determinadas tarefas, sendo estas geralmente caracterizadas por responsáveis diretos (R), participantes da execução e decisão daquela etapa (P) e pessoas que deveriam ser informadas das decisões obtidas (1).

0 preenchimento da matriz é realizado pela alta gerência, que tem a função de gestão do processo de desenvolvimento. A designação da participação nas etapas envolvidas deve ser pertinente ao envolvimento dos setores no processo de desenvolvimento.

\subsection{Organização das etapas do PDP}

Uma vez entendido como a empresa atualmente conduz o PDP, o próximo passo é a adequação das etapas atuais de desenvolvimento ao modelo 


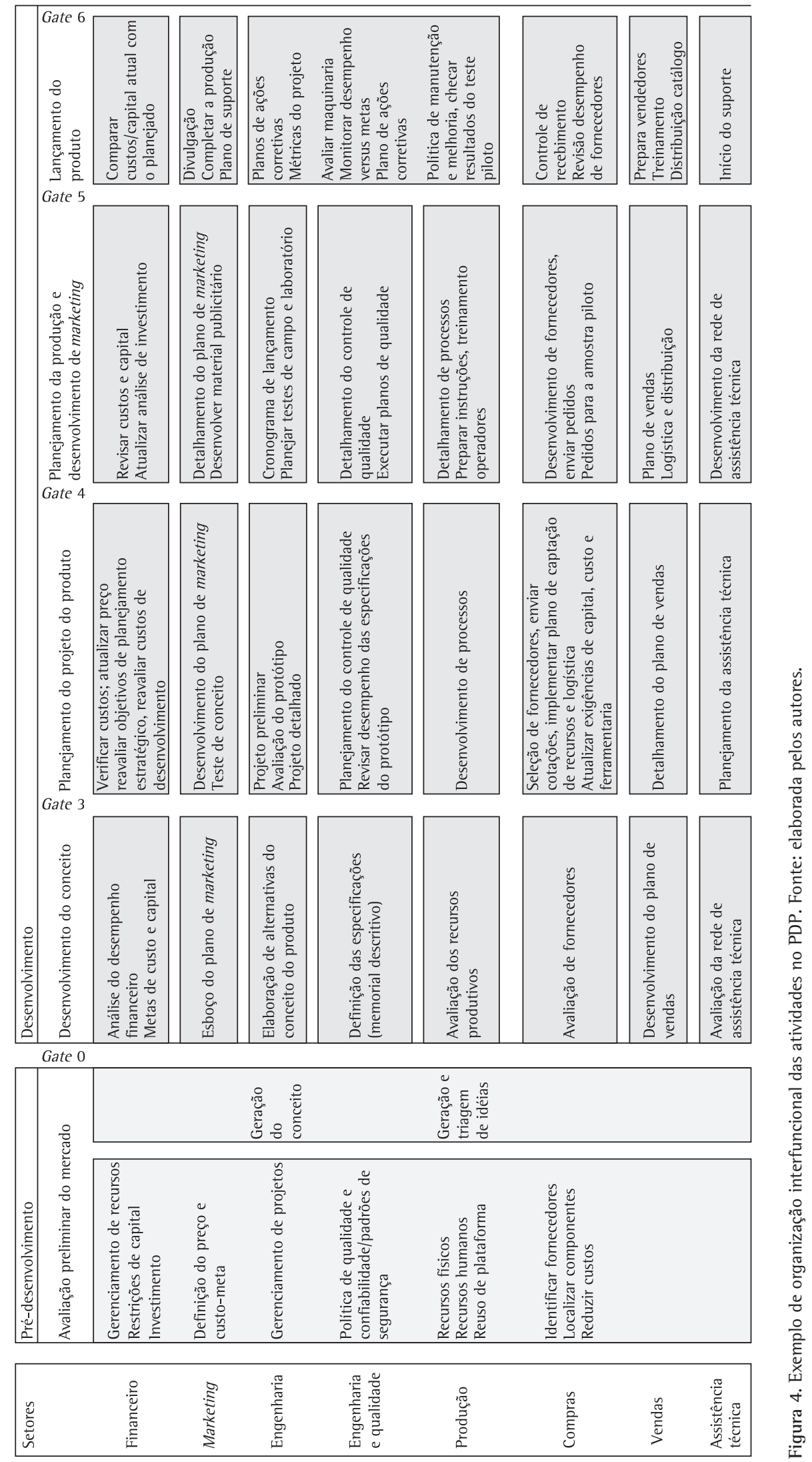


Quadro 3. Exemplo de matriz de responsabilidades para as fases do PDP.

\begin{tabular}{|c|c|c|c|c|c|c|c|c|}
\hline \multirow[b]{2}{*}{ Fases do PDP } & \multicolumn{8}{|c|}{ Setores envolvidos } \\
\hline & $\begin{array}{c}\text { Alta } \\
\text { gerência }\end{array}$ & Financeiro & Marketing & Produção & Projeto & Engenharia & Compras & Vendas \\
\hline $\begin{array}{c}\text { Avaliação preliminar } \\
\text { do mercado }\end{array}$ & $\mathrm{R}$ & $P$ & $P$ & - & 1 & - & - & - \\
\hline $\begin{array}{l}\text { Geração de } \\
\text { conceito }\end{array}$ & $\mathrm{R}$ & - & $P$ & - & $P$ & - & - & - \\
\hline $\begin{array}{c}\text { Geração e triagem } \\
\text { de ideias }\end{array}$ & $P$ & $P$ & $\mathrm{R}$ & $P$ & $P$ & - & - & - \\
\hline $\begin{array}{l}\text { Desenvolvimento } \\
\text { do conceito }\end{array}$ & - & - & $\mathrm{R}$ & $P$ & $P$ & - & 1 & 1 \\
\hline $\begin{array}{l}\text { Desenvolvimento do } \\
\text { projeto do produto }\end{array}$ & - & - & 1 & $P$ & $\mathrm{R}$ & $P$ & $P$ & - \\
\hline $\begin{array}{c}\text { Planejamento de } \\
\text { marketing/produção }\end{array}$ & - & - & $\mathrm{R}$ & $\mathrm{R}$ & $P$ & - & 1 & $P$ \\
\hline $\begin{array}{l}\text { Desenvolvimento } \\
\text { da produção }\end{array}$ & - & - & - & $\mathrm{R}$ & $P$ & $P$ & - & 1 \\
\hline $\begin{array}{l}\text { Lançamento } \\
\text { do produto }\end{array}$ & $\mathrm{P}$ & $\mathrm{P}$ & $\mathrm{R}$ & $\mathrm{P}$ & $P$ & $\mathrm{P}$ & $P$ & $\mathrm{P}$ \\
\hline
\end{tabular}

Fonte: elaborado pelos autores.

referencial adotado para integração das atividades. A apresentação das atividades do PDP será subdividida em etapas: pré-desenvolvimento, desenvolvimento e pós-desenvolvimento.

0 pré-desenvolvimento inicia na identificação e no reconhecimento de uma oportunidade de mercado e estende-se até a aprovação dos projetos que serão levados adiante. Fazem parte dessa fase a determinação das diretrizes de novos produtos, a geração de ideias, a triagem delas e a geração do conceito para cada ideia selecionada. Esse conceito é estabelecido de forma a registrar características gerais do produto a ser lançado, isto é, características capazes de traduzir a oportunidade do produto para dar início ao seu desenvolvimento. A fase de pré-desenvolvimento termina com o atestado de viabilidade do projeto. Cada projeto torna-se único e passa pelas fases de desenvolvimento e pós-desenvolvimento.

0 desenvolvimento transforma as ideias (conceito) em um produto físico. Começa com o desenvolvimento do conceito e definição dos principais parâmetros de projeto e segue até a liberação para a venda. Nessa fase são realizados, paralelamente, o desenvolvimento do protótipo, o de marketing e o de produção.

A organização de cada etapa pode ser apresentada em forma de descrição das atividades de execução, avaliação e documentação, conforme ilustrado no Quadro 4.

A organização das atividades está representada no modelo da Figura 3 e exemplificada no Quadro 4. De forma simplificada, os autores sugerem um roteiro, apresentado na sequência, em forma de etapas a serem vencidas na organização das atividades do PDP: (i) levantamento das principais situações de conflito (problemas relacionados ao PDP); (ii) escolher um modelo referencial na literatura para base da implementação; (iii) definir as fases gerais do Processo de Desenvolvimento de Produtos praticadas atualmente pela empresa e (iv) comparar o modelo referencial com o modelo praticado na empresa. Relacionar as atividades que devem ser (re)organizadas e implantadas seguindo os passos:

(a) Detalhar as principais atividades e relacionar com as grandes áreas;

(b) Relacionar as atividades com os departamentos envolvidos (matriz de responsabilidades);

(c) Mapear a ordem temporal das atividades: atividades dependentes que inciam após a conclusão ou alguma informação disponível e atividades independentes que poderiam ser antecipadas e em que momento isso aconteceria;

(d) Estabelecer pontos críticos (gates), pontos onde o projeto pode ser reavaliado, etapas que não foram realizadas corretamente, que poderiam comprometer a qualidade e custo do produto; etapas que coincidem com liberação de recursos; e

(e) Determinar documentos de comunicação interna e documentos resultantes das atividades realizadas.

Na sequencia, (v) gerar um documento (manual de procedimentos) de formalização do PDP: detalhamento das fases; (vi) executar treinamentos específicos para o líder do projeto e treinamento para as áreas envolvidas; (vii) estabelecer uma 


\begin{tabular}{|c|c|c|c|c|c|c|c|}
\hline & 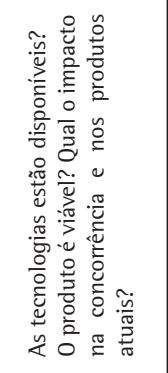 & 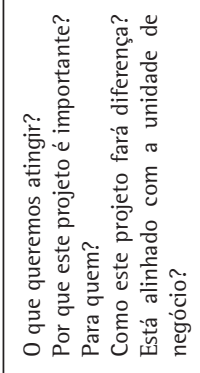 & 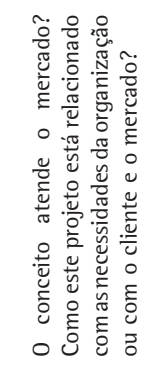 & 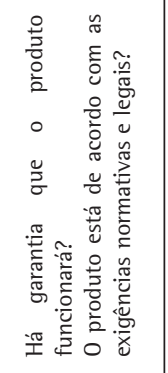 & 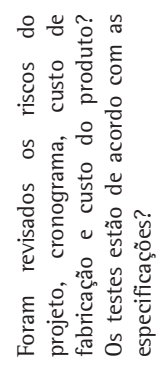 & 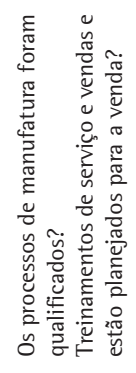 & 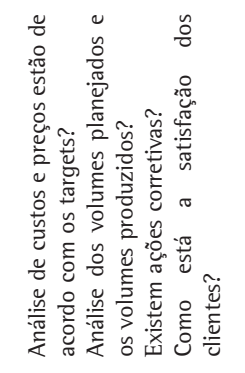 \\
\hline 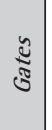 & 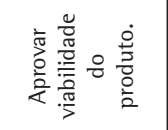 & 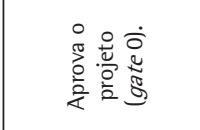 & 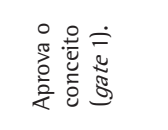 & 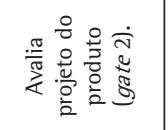 & 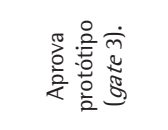 & 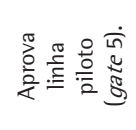 & 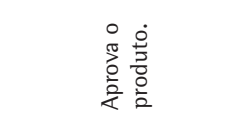 \\
\hline 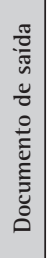 & 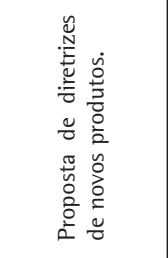 & 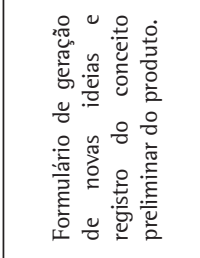 & 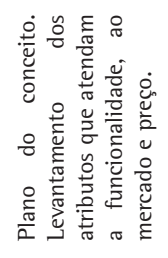 & 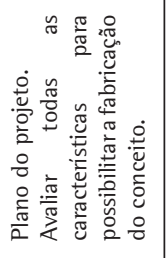 & 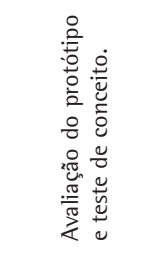 & 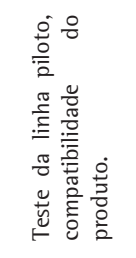 & 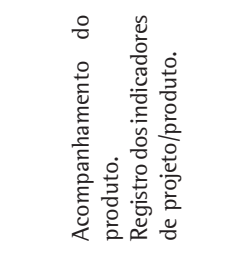 \\
\hline 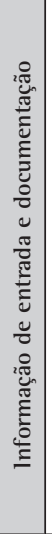 & 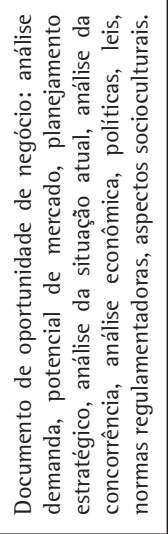 & 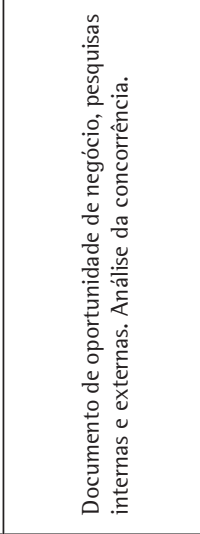 & 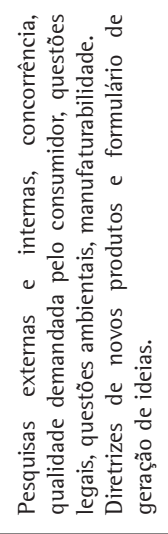 & 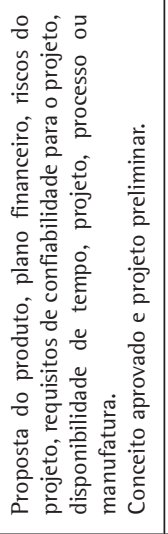 & 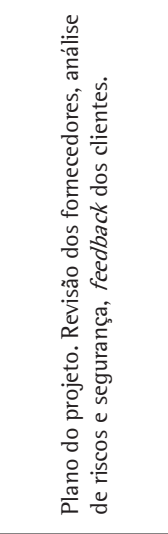 & 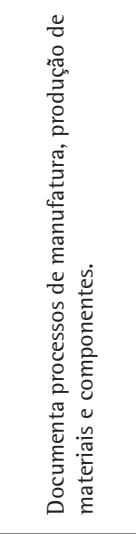 & 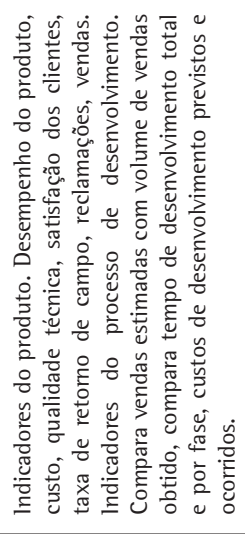 \\
\hline 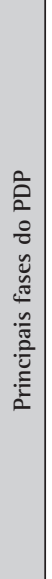 & 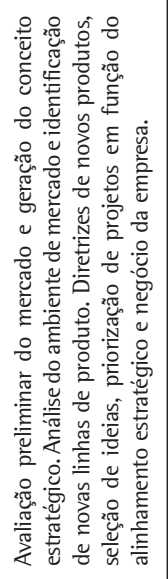 & 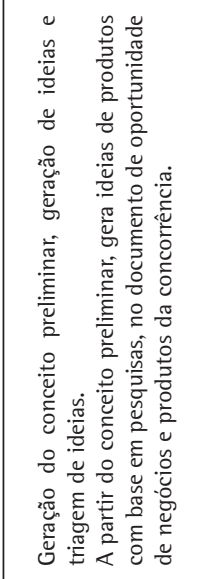 & 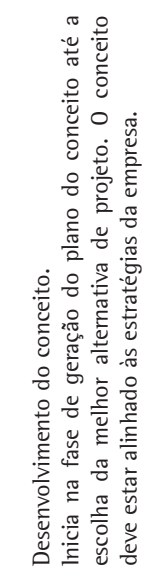 & 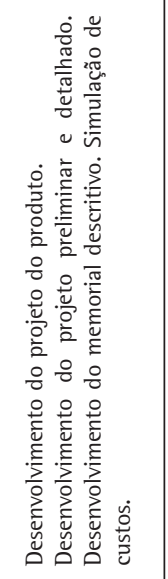 & 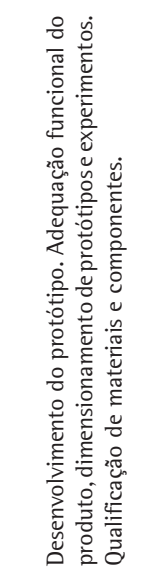 & 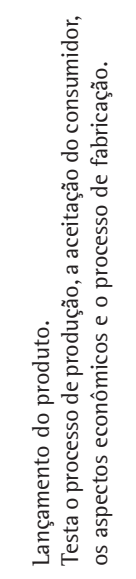 & 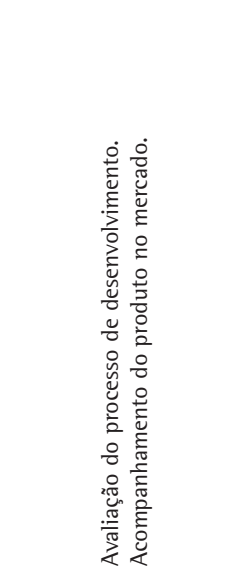 \\
\hline & & 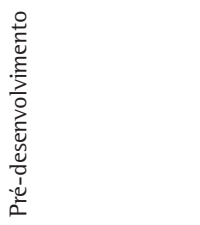 & \multicolumn{4}{|c|}{ 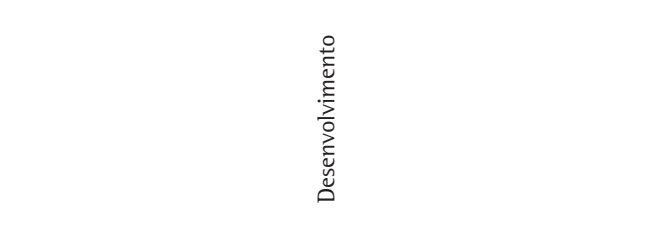 } & 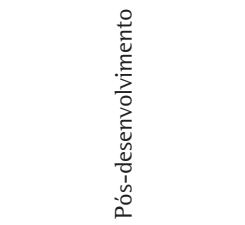 \\
\hline
\end{tabular}


política de acompanhamento do novo processo de desenvolvimento e (viii) estipular indicadores de acompanhamento do projeto e registro de melhorias que aperfeiçoem o modelo de desenvolvimento.

Esse roteiro é geralmente realizado com a equipe de denvolvimento de produto que coordena a gestão, desde o desenvolvimento do conceito até o lançamento das primeiras unidades.

\section{Considerações finais}

A organização do PDP da empresa contribui para diminuir tempos de ciclo de desenvolvimento e incidência de retrabalho, podendo auxiliar amplamente na obtenção de vantagens competitivas, sobretudo num mercado onde novas ideias lançadas em menor tempo têm maiores chances de sucesso. As empresas têm consciência de que processos padronizados conduzem mais eficientemente aos objetivos traçados no planejamento estratégico do produto. No entanto, a maioria das empresas não tem seus processos estruturados e trata o desenvolvimento como um departamento de responsabilidade única da engenharia.

Da mesma forma, nos últimos anos, muitos modelos de desenvolvimento de produtos surgiram, destacando diferentes fases e diferentes subetapas. Os modelos propostos por estudiosos da área incorporaram as fases de planejamento estratégico e marketing, antes e após o desenvolvimento de produto, para conduzir mais eficientemente as estratégias da empresa alinhadas ao desenvolvimento. Apesar da extensa literatura referente ao Processo de Desenvolvimento de Produtos, há dificuldade de adaptação dos modelos teóricos à execução prática nas empresas. Principalmente em empresas de pequeno e médio porte, onde se observa maior dificuldade na organização das atividades do PDP e posterior acompanhamento de atividades planejadas. Esse cenário se deve, em parte, à dificuldade de conduzir atividades periódicas de melhoria pela própria equipe de desenvolvimento sem a participação de uma equipe externa. Uma metodologia de solução de problemas adaptada ao PDP não foi encontrada na literatura que se concentra em métodos orientados a grandes empresas ou trata de projetos de melhoria como grandes projetos de transformação do PDP.

A experiência dos autores deste artigo, baseada no acompanhamento de empresas e na reflexão teórica dos principais autores de DP, permite delinear alguns aspectos-chave para o sucesso da implementação. Para estruturação do PDP, propõem-se diretrizes gerais apresentadas na forma de um método de (re)organização das atividades. A estruturação é constituída de duas fases principais: uma de intervenção no processo atual da empresa e outra que objetiva a aplicação de um modelo referencial para a integração das atividades.

0 modelo de intervenção pretende estabelecer um caminho de transição entre a situação atual (procedimentos de desenvolvimento de produto utilizados pela empresa, sejam eles documentados ou não) e o modelo de organização que se quer propor (apoiado em um modelo referencial). Para tanto, sugere-se um método de organização das atividades que, por sua vez, contempla o mapeamento delas por setor e o sincronismo das atividades.

A aplicação da proposta apresentada neste artigo pode auxiliar as empresas a conduzirem a transformação necessária em seus processos de desenvolvimento de produto, contribuindo tanto no planejamento da mudança como no detalhamento da nova organização das atividades do PDP.

\section{Agradecimentos}

Os autores agradecem à CAPES e ao CNPq pela concessão de bolsas de pesquisa que contribuiram para a realização deste trabalho.

\section{Referências}

ANDREASEN, M. M.; HEIN, L. Integrated product development. New York: Springer-Verlag, 1987.

BAXTER, M. Projeto de produto: guia prático para o design de novos produtos. 2. ed. São Paulo: Edgard Blucher, 1998.

BRESSAN, C. L. Mudança Organizacional: uma visão gerencial. In: SEMINÁRIO DE GESTÃO DE NEGÓCIOS, 1., 2004, Curitiba. Anais... Curitiba: FAE, 2004. v. 1. Disponível em: $<$ http://www.fae.edu/publicacoes/pdf/art_cie/art_25. pdf>. Acesso em: 12 dez. 2005.

CLARK, K. B.; FUJIMOTO, T. Product development performance: strategy, organization, and management in the world auto industry. Boston: Harvard Business Scholl Press, 1991.

CLIFT, T. B.; VANDENBOSCH, M. B. Project complexity and efforts to reduce product development cycle time. Journal of Business Research, v. 45, p. 187-198, 1999.

COOPER, R. G. New products: the factors that drive success. International Marketing Review, v. 11, n. 1, p. 60-76, 1994.

COOPER, R. G.; KLEINSCHIMIDT, E. J. Determinants of timeliness in product development. Journal of Product Innovation Management, v.11, p. 381-396, 1994.

CRAWFORD, C. M.; DI BENEDETTO, C. A. New products management. 6. ed. Chicago: McGraw-Hill, 2000.

CROW, K. DRM associates. characterizing and improving the product development process. [S.l.]: NPD, 2001. Disponivel em: <http://www.npd-solutions.com/ pdprocess.html>. Acesso em: 24 jul. 2002. 
CUNHA, G. D. et al. A reference model to support introducing product lifecycle management. In: GONÇALVES, R. J.; JIANZHONG, C.; STEIGER-GARÇÃO, A. Concurrent Engineering: the vision for the future generation enhanced interoperable systems. Amsterdam: Balkema Publishers, 2003.

FONSECA, S. A.; KRUGLIANSKAS. Inovação em microempresas de setores tradicionais: estudos de casos em incubadoras brasileiras in tecnologia e inovação: experiência de gestão na micro e pequena empresa. São Paulo: PGT/ USP, 2002.

GRIFFIN, A. E; PAGE, A. L. An interim report on measuring product development success and failure. Journal of Product Innovation Management, v. 10, p. 291-398, 1993.

GRUNER, K. E.; HOMBURG, C. Does customer interaction enhance new product success? Journal of Business Research, v. 49, p. 1-14, 2000.

KOTLER, P. Administração de marketing: análise, planejamento, implementação e controle. 5. ed. São Paulo: Atlas, 1998.

MCADAM, R.; STEVENSON, P.; ARMSTRONG, G. Innovative change management in SMEs: beyond continuous improvement. Logistics Information management, v. 13, n. $3 ;$ p. $138,2000$.

PAHL, G.; BEITZ, W. Engineering design: a systematic approach. London: Springer, 1996.

PHILLIPS, R.; NEAILEY, K.; BROUGHTON, T. A. Comparative study of six stage-gate approaches to product development. Integrated Manufacturing Systems, v. 10, n. 5, p. 289-297, 1999.

PRASAD, B. Concurrent engineering fundamentals: integrated product development. New Jersey: Prentice Hall, 1997.
PROJECT MANAGEMENT INSTITUTE - PMI. PMBOK: Project Management Body of Knowledge. Versão 1.0. Belo Horizonte: PMl - MG, 2000. Disponível em: <www.pmsp. org.br/exe/educaçao/ pmbok.asp>. Acesso em: 24 maio 2002. Tradução livre.

RENTES, A. F. TransMeth: proposta de uma metodologia para condução de processos de transformação de empresas. 2000. 229 f. Tese (Doutorado) - Escola de Engenharia de São Carlos, Universidade de São Paulo, São Carlos, 2000.

ROOZENBURG, N. F.M; EEKELS, J. Product design fundamentals and methods. Nova Jersey: John Wiley \& Sons, 1995.

ROZENFELD, H. et al. Gestão de Desenvolvimento de produtos. uma referência para a melhoria de processo. São Paulo: Saraiva, 2006.

SERVIÇO BRASILEIRO DE APOIO ÀS MICRO E PEQUENAS EMPRESAS - SEBRAE. Critérios e conceitos para classificação de empresas. [S.1.]: SEBRAE, 1999. Disponível em: <http://www.sebrae.com.br/customizado/estudos-epesquisas/bia-97-criterios-para-classificacao-do-portede-empresas/BIA_97/integra_bia?ident_unico=97>. Acesso em: 23 nov. 2007.

STARBEK, M.; GRUM, J. Concurrent engineering in small companies. International Journal of Machine Tools \& Manufatures, v.42, p. 417-426, 2001.

WHEELWRIGHT, S. C.; CLARK, K. B. Revolutionizing product development quantum leaps in speed, efficiency, and quality. New York: Macmillan, 1992.

WOOD JUNIOR, T. (coord.). Mudança Organizacional: liderança; teoria do caos; recursos humanos; logística integrada; inovações gerenciais; cultura organizacional; arquitetura organizacional. 3. ed. São Paulo: Atlas, 2002.

\title{
Diagnosis and intervention in medium size companies: a proposal for (re)organization of activities in the Product Development Process
}

\begin{abstract}
The product development process (PDP) may be defined as the way a company translates ideas and opportunities into products and services. This process demands clearly established phases as well as people and resources management. In the last decade, many reference models for PDP were proposed, presenting improvements and new phases of development, covering aspects ranging from marketing analysis to product discontinuation. Nevertheless, with regard to PDP structuralization, most companies face difficulties in adapting their reality to models presented in the literature. Clearly, a transition function is required to overcome the gap between industrial practices and PDP theoretical models. Based on the literature, as well as on best practices observed in companies from different sectors, this paper presents a method for the structuralization of PDP activities. The proposal focuses on two specific elements: (i) a method for intervention and (ii) a method for activity organization. Together, they represent an effective alternative for medium size companies searching to improve their PDP organization.
\end{abstract}

\section{Keywords}

Industrial engineering. Product development. Functional integration. 